hep-th/0001044

UT-838

\title{
Tachyon condensation and Boundary States in Bosonic String
}

\author{
Y. Matsuo \\ Department of Physics, University of Tokyo \\ Hongo 7-3-1, Bunkyo-ku, Tokyo 113-0034, Japan
}

\begin{abstract}
We discuss tachyon configuration for the unoriented bosonic string theory which produces a bosonic string theory with $S O(32)$ gauge symmetry in ten dimensions. It is closely related to the tachyon condensation scenario proposed by A. Sen. We also give the boundary state description of the tachyon condensation process, with some emphasis on the rôle of orbifold conformal field theory.
\end{abstract}

1 Talk given at YITP workshop "Tachyon condensation and boundary state" (October 27-29, 1999) 


\section{Introduction}

After some intensive study carried out last year, (for example see [1] ), it becomes now evident that there are several motivations to study tachyon condensation scenario. For example, (i) To seek correct vacuum of modular invariant but tachyonic string theories, for example in bosonic strings, type 0 (OA, OB), and also some heterotic strings. (ii) To find stable non-SUSY soliton. Particularly successful examples are the construction of the type I spinor particle [2], and also non-SUSY string junction [3]. (iii) To find unstable anomaly free string theories [4]. Some examples are $U(N) \times U(N)$ type IIB superstring theory, $U(N)$ type IIA, and type I analogue. This is closely related to Witten's K-theory argument [5] since in his approach arbitrary D-brane is constructed out of several pairs of $D-9$ and $\bar{D}-9$ branes. Such a $D-9$ configuration gives rise to the extra gauge symmetry in those theories.

Tachyon condensation is a dynamical process like Higgs mechanism. At this moment, although there are many important references [6], there are still many misteries in our understanding in terms of conformal field theory. In this paper, we choose a particulary simple example (bosonic string) and demonstrate the deformation in terms of boundary conformal field theory explicitly. We point out that the duality in conformal field theories play essential rôle to understand the deformation.

\section{A brief review of tachyon condensation}

Consider $D-p-\bar{D}-p$ system. Open string sectors are labeled by $U(2)$ CP factor. Fermion number operator for CP factor becomes nontrivial.

$$
(-1)_{C P}^{F}=\left(\begin{array}{cc}
1 & 0 \\
0 & -1
\end{array}\right)\left(=\sigma_{3}\right)
$$

For the total open string wave function $\Psi^{\text {total }}=\Psi^{o s c} \otimes \Psi^{C P}$, the fermion number parity operator acts as,

$$
(-1)^{F} \Psi^{\text {total }}=\left((-1)_{o s c}^{F} \Psi^{o s c}\right) \otimes\left(\sigma_{3} \Psi^{C P} \sigma_{3}\right)
$$

It implies that:

(i) In $\Psi_{C P}=\sigma_{0}(=1), \sigma_{3}$ sector, we have the usual GSO projection. There are $U(1)_{\sigma_{0}} \times U(1)_{\sigma_{3}}$ gauge fields at the massless level.

(ii) In $\Psi_{C P}=\sigma_{1}, \sigma_{2}$ sector, we have the opposite GSO projection which gives a complex tachyon field $(T, \bar{T})$.

We remark that $T$ (resp. $\bar{T}$ ) have charge $2(-2)$ under $A_{\sigma_{3}}$ but neutral under $A_{\sigma_{0}}$. We assume that the tachyon field has the following potential which is analogous to that of Higgs fields. Based on this potential we may discuss the 
process of the pair annihilation of the D-branes in the similar fashion with more familiar symmetry breaking mechanism?.

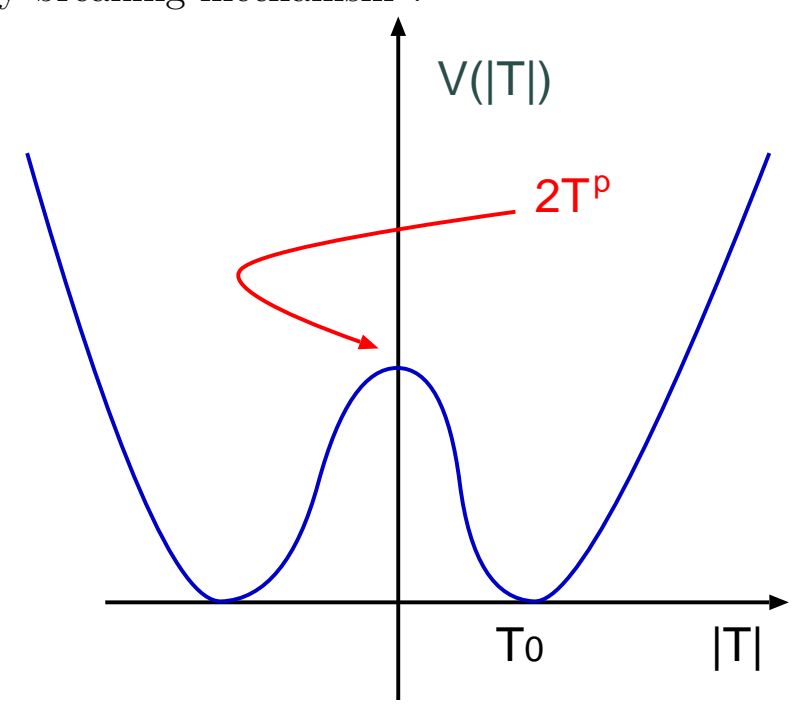

There are two equivalent scenarios to realize the tachyon condensation. Let us start from $D-(p+2)$ and $\bar{D}-(p+2)$ branes.

- Step by step method proposed by A. Sen [1]:

1. First consider kink configuration for complex tachyon $\left(T(x) \rightarrow \pm T_{0}\right.$ as $x \rightarrow \pm \infty)$ which define $\mathrm{D}$ - $(p+1)$ brane.

2. Since moduli of tachyon is defined by $S^{1}$, such configuration is topologically unstable.

3. On this $\mathrm{D}-(p+1)$ brane, there is a real-valued tachyon field. One may again consider a kink configuration, which defines $D-p$ brane.

4. Since the moduli is $Z_{2}$, this tachyon configuration becomes topologically stable which defines $D-p$ brane.

- Topological method proposed by E. Witten [5]:

We consider a topologically nontrivial configuration (vortex) for gauge fields and tachyons in two dimensions,

$$
\begin{aligned}
\frac{1}{2 \pi} \int F_{\sigma_{3}} & =n \quad \frac{1}{2 \pi} \int F_{\sigma_{0}}=0 \\
T(x) & \sim e^{i n \theta}\left|T_{0}\right| \quad \text { as } \quad|x| \rightarrow \infty \\
& \sim x_{1} \sigma_{1}+x_{2} \sigma_{2}+O\left(|x|^{2}\right) .
\end{aligned}
$$

D- $p$ and D- $\bar{p}$ can be pair annihilated if tachyon field takes $|T|=\left|T_{0}\right|$ at spacial infinity. Then $\mathrm{n} \mathrm{D}-(p-2)$ branes appear at the core of vorticity.

\footnotetext{
${ }^{2}$ Recently there are some development to prove it by using the string field theory [7].
} 


$$
U(1)_{\sigma_{0}} \times U(1)_{\sigma_{3}} \text { is broken to } U(1)_{\sigma_{0}} \cdot \text { ? }^{3}
$$

BCFT method is easier to be applied to the first scenario. On the other hand, its topological nature is illuminated in the second. We will use both of them.

\section{A Scenario for Bosonic string}

In the following sections, we will mainly consider the tachyon condensation of the bosonic string by following [9]. Some of the key motivations are (i) it is the simplest string models, and (ii) it is nonetheless the most generic string theory. It is supposed to contain superstring theories, type 0 theories, heterotic strings as its particular vacuum. It is therefore important to seek the fate of the bosonic string if any. It would be also desirable if one may find a dynamical scenario for the compactification of the 26 dimensional space-time.

A possible candidate of the destination of the open bosonic string would be,

1. $S O(32)$ type I string

2. Type 0 string $(S O(32) \times S O(32)$ theory) which is more plausible since it has bosonic spectrum and still contains a closed string tachyon.

Open unoriented bosonic string was studied by some groups [10], and it has been known that a consistent model exists with $D=26$ bosonic string and space filling $D$-25-branes. Tadpole free condition restricts the number of 25-branes to be $2^{13}=8192$. This condition comes from the cancellation of massless excitation arising from the boundary states of the open boundary and the crosscap. If this condition is satisfied, the model becomes a finite theory if one applies the zeta function regularization to the infinity from the tachyon mode.

Although it is very interesting that such a consistent model exists in purely bosonic theory, it is obviously discouraging that

1. it has a huge gauge symmetry $S O(8192)$ to be realistic,

2. it is defined only in 26 dimensions,

3. it has many open string tachyons since there is no GSO projection,

4. there is no D-brane charges and every D-branes becomes unstable.

In the following discussions, we would like to point out that these weak points may be eliminated by using a topologically nontrivial configuration for the tachyon fields.

\footnotetext{
${ }^{3}$ Fate of $U(1)_{\sigma_{0}}$ is known to be Witten's U(1) problem. See for example $[\mathbb{8}]$ for the attempts to understand the issue.
} 


\subsection{Sen's argument}

Sen [11] applied applied his step by step method to the system with two D- $(p+1)$ branes. There are four tachyons arising from four CP sectors of the open string. One may introduce $Z_{2}$ Wilson line to one of the D-branes to make the intertwining open string anti-periodic in one space direction. One D-p-brane will appear at the position of the kink of the tachyon field, f while original two D-branes disappear. In the exact treatment of such process, one need to compactify one of the space direction on a circle. By such compactification, the mass squared of tachyon field increases while the radius of $S^{1}$ become smaller. At a specific radius, the tachyon mode becomes massless and the exact treatment of the tachyon condensation becomes possible. In this process, one is forced to reduce the number of the uncompactified dimensions. Successive application of this idea to $S O\left(2^{13}\right)$ theory would imply that one $D-12$ brane will be produced at the end of tachyon condensation while we have 13 space-time dimensions. Since such a theory can not be tadpole free in any respect, this scenario seems rather unnatural.

Tachyon appear in interwinning mode Tachyon kink describes new D-brane
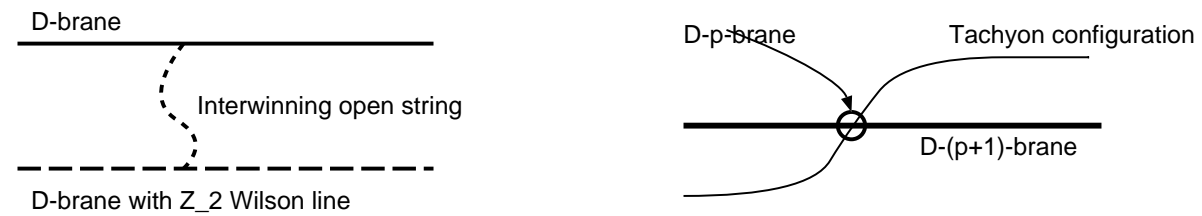

Sen have also argued that there are other options.

- Starting from a single D-brane, there exists an open string tachyon. If one consider the kink for this tachyon mode, one $\mathrm{D}-(p+1)$-brane may produce several D-p-branes.

- If tachyon develops several kinks, there may appear several D-p-branes. Therefore, the number of D-branes can be arbitrary.

The first possibility seems not plausible since Cardy's constraint [12 on the boundary state implies that it is not possible to have an anti-periodic tachyon field in case of a single D-brane. The second possibility seems rather artificial. Such an arbitrariness always arises if we seek the tachyon condensation in topologically unstable configurations. However, as wee see, one may achieve some kind of topological stability even in the case of bosonic string. If we restrict our scope to the topologically stable sectors, there is no such arbitrariness.

\subsection{Our proposal}

It seems to us that the only topologically stable tachyon condensation scenario is a process where two $\mathrm{D}$ - $(p+2)$-branes with tachyon condensation produce a

\footnotetext{
${ }^{4}$ Exact description in terms of boundary state will be discussed in the next section.
} 
single D- $p$ brane while compactifying two space dimensions toroidally. In this scenario, we have $2^{13-d / 2}$ D-branes while compactifying $d$ dimensions. It matches with the tadpole free conditions for generic open string. If we take $d=16$, we have $S O(32)$ theory at dimension 10 which is a candidate model which can be dual to type I or type 0 string theories.

To explain the topological nature, let us consider two D-branes. The gauge group associated with it is $S U(2)$ except for the overall $U(1)$. The vacuum moduli of the tachyon potential is $S^{3}$. In such a situation, one may not expect topologically stable configuration since $\pi_{1}\left(S^{3}\right)=0$.

However, at the special infinity, the gauge transformation can be nontrivial. We recall that the open string transforms in adjoint representation. The actual gauge group which acts nontrivially on the open string is not $S U(2)$ but $S O(3)$. In this context one may have a topologically nontrivial loop in the gauge configuration space since,

$$
\pi_{1}(S O(3))=\mathbf{Z}_{2}
$$

It also means that the topologically nontrivial sector is in a sense unique. One may interpret it that the number of the D-p brane which will appear at the core is restricted to be one.

To illustrate this idea more explicitly, we compactify two dimensions toroidally and require the behavior of the tachyon field at the core of vorticity,

$$
T(x) \sim x^{1} \sigma^{1}+x^{2} \sigma^{2} \quad(\text { as }|x| \rightarrow 0) .
$$

Tachyon must be anti-periodic in $x^{i}$ directions in $\sigma^{i}$ sector. This configuration appeared in [5] to describe nontrivial vortex configuration.

Roughly speaking, such a configuration is realized if we introduce a kind of non-abelian Wilson lines which produces curvature,

$$
A_{1} \propto \sigma_{2} \quad A_{2} \propto \sigma_{1}
$$

It costs some energy but since they turn out to be in the topologically nontrivial, such a configuration is stable. A simple realization is possible if we impose the following boundary condition for the open string,

$$
\begin{aligned}
& \Psi\left(x^{1}+2 \pi R, x^{2}\right)=\sigma_{2} \Psi\left(x^{1}, x^{2}\right) \sigma_{2}, \\
& \Psi\left(x^{1}, x^{2}+2 \pi R\right)=\sigma_{1} \Psi\left(x^{1}, x^{2}\right) \sigma_{1} .
\end{aligned}
$$

It belongs to the topologically nontrivial sector since the gauge transformation (=Wilson line) around the loop is $\sigma^{1} \sigma^{2}\left(\sigma^{1}\right)^{-1}\left(\sigma^{2}\right)^{-1}=-1$ p. We summarize the periodicity along each direction,

\footnotetext{
${ }^{5}$ This is a projective representation of the lattice translation group. Similar nontrivial representation in $\mathrm{CP}$ appeared in the description of the discrete torsion in orbifold conformal field theory 13.
} 


\begin{tabular}{|c||c|c|c|c|}
\hline & $\sigma^{0}$ & $\sigma^{1}$ & $\sigma^{2}$ & $\sigma^{3}$ \\
\hline \hline$x^{1}$ & + & - & + & - \\
$x^{2}$ & + & + & - & - \\
\hline
\end{tabular}

Gauge symmetry is broken from $U(2)$ to $U(1)$ which is quite unusual but necessary to describe pair annihilation of two D-branes.

Since we simply impose the antiperiodic boundary condition to some components of the open string, the spectrum of the topologically nontrivial sector is very easy to calculate. If the original open string lives in radius $R$, the momentum distribution of the twisted theory is the equally separated momenta with the separation $1 / 2 R$. Therefore, the spectrum of the topologically nontrivial sector becomes identical the either of the following two systems,

1. one $\mathrm{D}-(p+2)$ brane in radius $2 R$,

2. one D- $p$ brane in radius $1 / 2 R$.

It supports our expectation that only one D- $p$ brane appear at the core of vorticity.

To define the unoriented string theory, we need to impose the orientation projection $\Omega$ to the open string wave function. At each mass level, we have project out either symmetric (anti-symmetric) part of the wave function. Such the projection is compatible with our boundary condition since Pauli matrices satisfies well-defined parity under adjoint action of $\Omega$. The symmetry breaking pattern $U(2) \rightarrow U(1))$ remains the same but the precise correspondence in the momentum distribution is corrupted.

To extend our analysis to the compactification of the higher dimensional tori is straightforward. To have nontrivial configuration in $2 d$ dimensions, general prescription will be,

$$
\begin{aligned}
& \Psi\left(x^{26-2 d}, \cdots, x^{25-2 d+i}+2 \pi R, \cdots, x^{25}\right) \\
& \quad=\tilde{\Gamma}_{i} \Psi\left(x^{26-2 d}, \cdots, x^{25-2 d+i}, \cdots, x^{25}\right) \tilde{\Gamma}_{i}
\end{aligned}
$$

where $\tilde{\Gamma}$ satisfies,

$$
\begin{aligned}
& \left\{\tilde{\Gamma}_{i}, \tilde{\Gamma}_{j}\right\}=2 \delta_{i j} \\
& \left\{\tilde{\Gamma}_{i}, \Gamma_{i}\right\}=0 \quad\left[\tilde{\Gamma}_{i}, \Gamma_{j}\right]=0 \quad(i \neq j)
\end{aligned}
$$

With this prescription, $\Psi$ behaves at the core as,

$$
\Psi(x)=\sum_{i=1}^{2 d} x^{25-2 d+i} \Gamma_{i}+O\left(|x|^{2}\right)
$$

which is identical to the tachyon configuration of the superstring as discussed by Witten [5]. With this boundary condition, Chan-Paton gauge symmetry is broken from $U\left(2^{d}\right)$ to $U(1)$. 


\subsection{Symmetry enhancement from closed strings?}

At $R=1 / \sqrt{2}$, we get extra gauge symmetry from vertex operator $e^{i X(z) / R}$ in the closed string sector. In general, if the compactified direction is given by the root lattice of $G$, gauge group is enhanced to $G$. If we compactify 16 dimensions, the maximal enhancement is given by $S O(32)$ or $E_{8} \times E_{8}$. This well-known mechanism was used to construct the heterotic string theory.

Tachyon fields which interpolates the different D-branes becomes anti-periodic in the compactified direction and it has the mode expansion,

$$
T(x)=\sum_{n \in \mathbf{Z}} T_{n-1 / 2} e^{i(n-1 / 2) X} .
$$

Mass of each mode $T_{n-1 / 2}$ is given by $m^{2}=-1+(n-1 / 2)^{2} / 2 R^{2}$. The lowest mode $T_{ \pm 1 / 2}$ becomes massless when $R=1 / 2 \sqrt{2}$. We expect that the existence of massless mode signifies the enhanced gauge symmetry. However the radius for the massless tachyon is half of that of the closed string gauge enhancement point.

\section{Tachyon condensation and Boundary CFT}

As we saw in the last section, if we fix the radius of the target space to $R=1 / 2 \sqrt{2}$, some of the tachyon mode becomes massless. In the case of the closed string excitation, such a massless mode is used to deform the background (metric, antisymmetric tensors etc.) of the target space. On the other hand, the deformation by the massless open string mode usually triggers the deformation of the D-brane to which open string is attached. In our situation, the deformation by massless mode induces the tachyon condensation and we want to follow the change of the D-brane state in such a process as exactly as possible. In this section, we give an explicit description of boundary conformal field theory in Sen's scenario.

Before we discuss the detail, it is useful to to indicate that the radius $R=$ $1 / 2 \sqrt{2}$ is exactly the point where the moduli space of toroidal compactification and orbifold compactification meets. This point is quite essential in the following discussion. 


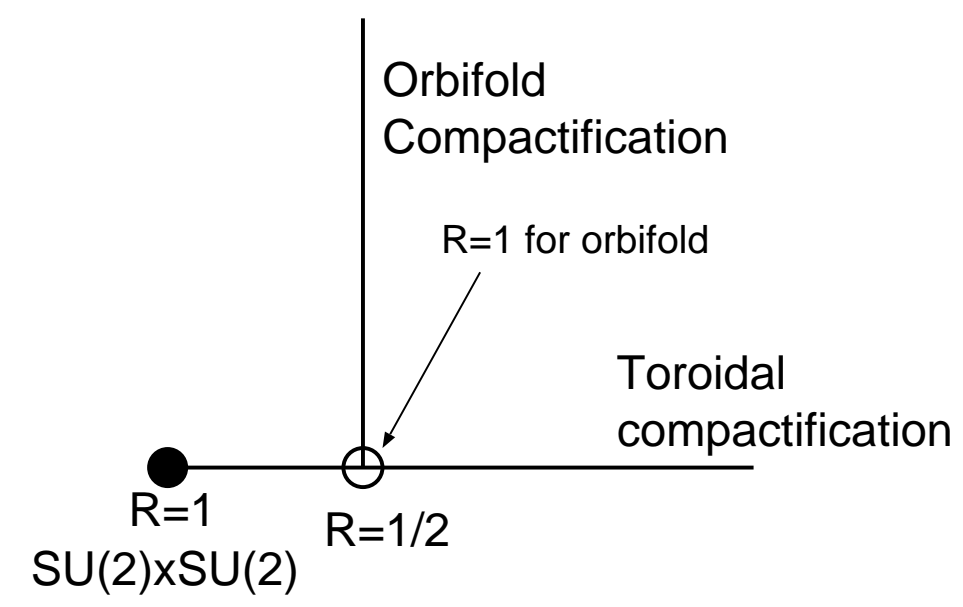

\subsection{Analysis in open string Hilbert space}

In this section, we review Sen's discussion [11] of the analysis which uses the open string Hilbert space. To treat the condensation of the tachyon field, Sen introduced new variables to describe the compactified direction $X$,

$$
\begin{aligned}
e^{i \sqrt{2} X} & =\partial Y+i \partial Z \\
e^{i \sqrt{2} Y} & =\partial X-i \partial Z \\
e^{i \sqrt{2} Z} & =\partial X+i \partial Y
\end{aligned}
$$

By using the new variables, tachyon condensation is equivalent to the deformation induced by $\oint \partial Y \otimes \sigma_{1} d z$.

As we emphasized before, the radius for the massless tachyon is not equal to the radius for the gauge enhancement in the closed string sector. Although we introduced the variables of $S U(2)$ current algebra, we need to project out some part of the spectrum. We should not forget that $e^{ \pm i \sqrt{2} X}$ is antiperiodic in direction $X$.

Such a projection can be carried out by introducing two parity operators,

$$
\begin{aligned}
h & \equiv X \rightarrow X+\pi / \sqrt{2} \\
g & \equiv X \rightarrow-X
\end{aligned}
$$

The first one is the translation in $X$ direction. Open string modes in $\sigma^{1}, \sigma^{2}$ sectors becomes odd under this operator.

On the other hand, we do not originally have any projection of the second parity operator. However, to define a similar projection in $Y$ variable, these two operators change their rôle,

$$
h_{X}=g_{Y} \quad g_{X}=h_{Y} .
$$

This relation motivates us to introduce the second operator. 
Let us now proceed to define necessary projections in terms of $Y$ variable. Originally in $Y$ direction, we have both boundary condition

$$
\Psi(Y+2 \pi) \equiv g \Psi(Y)= \pm \Psi(Y) .
$$

Since we have both periodic and anti-periodic boundary conditions, it is more natural to extend periodicity in $4 \pi$.

$$
\Psi(Y+4 \pi)=\Psi(Y)
$$

By the tachyon condensation deformation in $Y$ direction, we deform this boundary condition to

$$
\Psi(Y+4 \pi)=\sigma_{1} \Psi(Y) \sigma_{1}
$$

The eigenvalues of $h$ and $g$ in each sectors are now deformed. We summarize it in the next table,

\begin{tabular}{|c||c|c|}
\hline & $h_{Y}=g_{X}$ & $g_{Y}=h_{X}$ \\
\hline \hline$I$ & \pm 1 & + \\
$\sigma_{1}$ & \pm 1 & - \\
$\sigma_{2}$ & $\pm i$ & - \\
$\sigma_{3}$ & $\pm i$ & + \\
\hline
\end{tabular}

If we combine the four sectors, we have both signs in $g_{Y}$ parity. For each sign, we have four twists $\pm 1, \pm i$ in the boundary condition. This indicates that momentum is now quantized in the unit $1 / 4$ i.e. the compactification radius is apparently changed to $\sqrt{2}$. If we take the T-dual in $Y$ direction, Radius becomes $1 / 2 \sqrt{2}$ - original radius and two $\mathrm{D}(p+1)$-branes become single $p$-brane.

\subsection{Tachyon condensation in Boundary state}

We now turn back to describe this tachyon condensation process in terms of the boundary state. The deformation is induced by insertions of

$$
\exp \left(i \varphi \oint d z \partial Y \otimes \sigma_{1}\right)
$$

at the boundary.

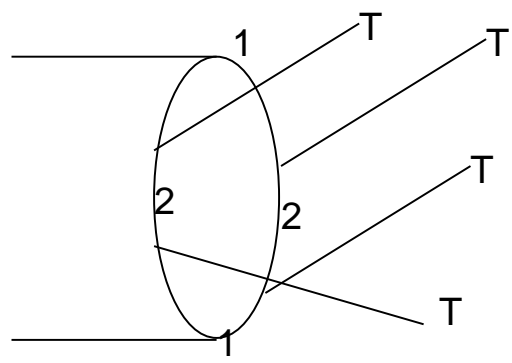


There are two difficult points which we need some care to discuss the deformation.

The first one is that the D-brane at the boundary must be switched by $\mathrm{CP}$ factor $\sigma_{1}$ at each insertion point as illustrated in the above figure. At each end of the inserted open string, we have different D-branes and we need to toggle CP factor in two ends.

The insertion of off-diagonal CP factor is usually treated by using the trace. In our case, we need some care since we have already deformed one of the D-brane by using $Z_{2}$ Wilson line.

The second point is that the deformation operator is the vertex operator in terms of original field, $\partial Y=\cos (X)$. We have to change the dynamical variable to $Y$ as in the open string approach. The $Z_{2}$ transformation $h$ act on $Y$ as $Y \rightarrow-Y$. It suggests that the natural framework is to use $Z_{2}$ orbifold variable.

To describe orbifold CFT, it is useful to prepare the boundary states for the theory with $S^{1}$ compactification. We have two types of boundary states,

- Dirichlet: $|D(\varphi)\rangle \quad(\varphi \sim \varphi+2 \pi r)$ $\frac{1}{\sqrt{2 r}} \sum_{k=0}^{\infty} e^{-i k \varphi / r} \exp \left(-\sum_{n=1}^{\infty} a_{n}^{\dagger} \widetilde{a}_{n}^{\dagger}\right)|0, k\rangle$

- Neumann: $|N(\widetilde{\varphi})\rangle \quad(\widetilde{\varphi} \sim \widetilde{\varphi}+\pi / r)$

$$
\sqrt{r} \sum_{w=0}^{\infty} e^{-2 i r w \widetilde{\varphi}} \exp \left(\sum_{n=1}^{\infty} a_{n}^{\dagger} \widetilde{a}_{n}^{\dagger}\right)|w, 0\rangle
$$

Physical interpretation of the deformation parameters are well-known to be location of D-brane for $\varphi$ and the Wilson line for $\widetilde{\varphi}$. In this language, the boundary state before tachyon condensation is

$$
|N(0)\rangle+|N(\pi / 2 r)\rangle .
$$

The boundary states for $S^{1} / Z_{2}$ orbifold was discussed in [14].

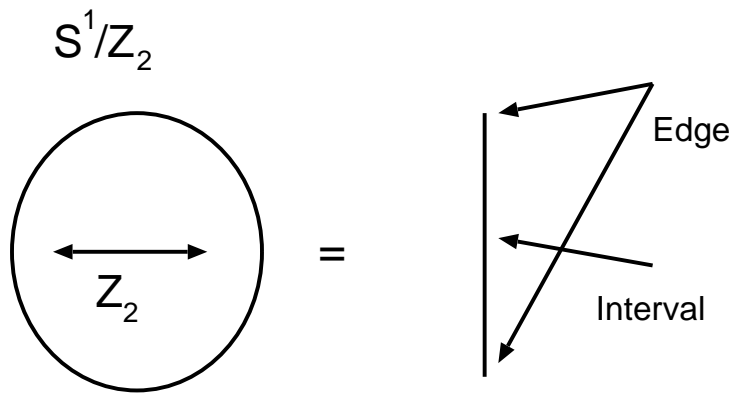

In this case, the boundary state which describes the location of D-brane in the interval and those located at the edges are essentially different. We write them by using the boundary states for $S^{1}$ compactification.

1. Boundary state for interval $(0<\varphi<\pi r, 0<\widetilde{\varphi}<\pi / 2 r)$

$$
\left|D_{O}(\varphi)\right\rangle=\frac{1}{\sqrt{2}}(|D(\varphi)\rangle+|D(-\varphi)\rangle)
$$




$$
\left|N_{O}(\varphi)\right\rangle=\frac{1}{\sqrt{2}}(|N(\varphi)\rangle+|N(-\varphi)\rangle)
$$

2. Boundary state for edges (half D-branes) $(\varphi=0, \pi r, \widetilde{\varphi}=0, \pi / 2 r)$ :

$$
\begin{aligned}
\left|D_{O}(\varphi) \pm\right\rangle & =\frac{1}{\sqrt{2}}|D(\varphi)\rangle \pm \frac{1}{2^{1 / 4}}\left|D(\varphi)_{T}\right\rangle \\
\left|N_{O}(\widetilde{\varphi}) \pm\right\rangle & =\frac{1}{\sqrt{2}}|N(\widetilde{\varphi})\rangle \pm \frac{1}{2^{1 / 4}}\left|N(\widetilde{\varphi})_{T}\right\rangle
\end{aligned}
$$

Open string partition function with specific $Z_{2} \times Z_{2}$ charge (generated by $h$ and $g$ ) can be written in terms of edge boundary states:

$$
\begin{aligned}
& Z_{++}=\frac{1}{2} \frac{\sum_{n} q^{4 n^{2}}}{\prod_{n}\left(1-q^{n}\right)}+\frac{1}{2} \frac{1}{\prod_{n}\left(1+q^{n}\right)}=\left\langle N_{O}\left(\widetilde{\varphi}_{ \pm}\right) \pm|\Delta| N_{O}\left(\widetilde{\varphi}_{ \pm}\right) \pm\right\rangle \\
& Z_{+-}=\frac{1}{2} \frac{\sum_{n} q^{4 n^{2}}}{\prod_{n}\left(1-q^{n}\right)}-\frac{1}{2} \frac{1}{\prod_{n}\left(1+q^{n}\right)}=\left\langle N_{O}\left(\widetilde{\varphi}_{ \pm}\right) \pm|\Delta| N_{O}\left(\widetilde{\varphi}_{ \pm}\right) \mp\right\rangle \\
& Z_{-+}=\frac{1}{2} \frac{\sum_{n} q^{4(n+1 / 2)^{2}}}{\prod_{n}\left(1-q^{n}\right)}=\left\langle N_{O}\left(\widetilde{\varphi}_{ \pm}\right) \pm|\Delta| N_{O}\left(\widetilde{\varphi}_{\mp}\right) \pm\right\rangle \\
& Z_{--}=\frac{1}{2} \frac{\sum_{n} q^{4(n+1 / 2)^{2}}}{\prod_{n}\left(1-q^{n}\right)}=\left\langle N_{O}\left(\widetilde{\varphi}_{ \pm}\right) \pm|\Delta| N_{O}\left(\widetilde{\varphi}_{\mp}\right) \mp\right\rangle
\end{aligned}
$$

where $\widetilde{\varphi}_{ \pm}=0, \pi / 2 r$.

By choosing specific radius $r=1 / 2 \sqrt{2}$, two $Z_{2}$ symmetry becomes interchangeable,

$$
Z_{+-}=Z_{-+}
$$

This accidental symmetry is a consequence of the fact that this model is an orbifold CFT and at the same time a CFT on $S^{1}$. In terms of edge boundary states, interchange of $h$ and $g$, (or $J_{3}$ and $J_{1}$ ) is described by the change of the rôle of two edges and the sign of the twisted sector, $\left|N_{O}\left(\widetilde{\varphi}_{\epsilon_{1}}\right) \epsilon_{2}\right\rangle \leftrightarrow\left|N_{O}\left(\widetilde{\varphi}_{\epsilon_{2}}\right) \epsilon_{1}\right\rangle$.

To summarize, the deformation associated with the tachyon condensation can be achieved by the following steps. (i) Prepare the boundary state which describe two bosonic D-branes with $Z_{2}$ Wilson line on one of them (2). (ii) Reinterpret the boundary state as those of $S_{1} / Z_{2}$ orbifold model. (iii) Change the rôle of $J_{1}$ and $J_{3}$. In terms of the boundary state, it amounts to the interchange of two parameters $\tilde{\varphi}$ and $\epsilon$. (iv) Deform the parameter $\tilde{\varphi}$ of one of the D-brane boundary states. More explicitly, it can be carried out as follows.

$$
\begin{aligned}
|N(0)\rangle+|N(\pi / 2 r)\rangle & =\sqrt{2} \sum_{\epsilon_{1}, \epsilon_{2}}\left|N_{O}\left(\widetilde{\varphi}_{\epsilon_{1}}\right) \epsilon_{2}\right\rangle \\
& =\sqrt{2} \sum_{\epsilon_{1}, \epsilon_{2}}\left|N_{O}\left(\widetilde{\varphi}_{\epsilon_{2}}\right) \epsilon_{1}\right\rangle \\
& \rightarrow \frac{1}{\sqrt{2}} \sum_{\epsilon_{1}, \epsilon_{2}}\left|N_{O}\left(\widetilde{\varphi}_{\epsilon_{2}}\right) \epsilon_{1}\right\rangle+\sqrt{2}\left|N_{O}\left(\frac{\pi}{\sqrt{2}}\right)\right\rangle
\end{aligned}
$$


We note that the boundary states in the final line produces the same partition function in the open string sector as that of $|D(0)\rangle$ for $S^{1}$ compactified model. This calculation explicitly demonstrates the production of one D- $p$ brane from two D- $(p+1)$ branes by the tachyon condensation. However, we have to mention one subtlety. In passing from the second to the third line, the intermediate state in the course of deformation can not be properly interpreted as D-brane boundary state since it would produce partition function with fractional coefficient in the open string sector. Namely, it does not satisfy Cardy's constraint. In this sense, one can not describe the tachyon condensation as the continuous deformation of the boundary state. This phenomena was also discussed by Sen and he explained it by showing the appearance tadpole. In this sense, it is more appropriate that two vacua (a) two D- $p+1$ brane system and (b) one D- $p$ brane system are not connected continuously but belong to the different topological sectors.

\section{Discussion}

In the second half of this paper, I discussed duality with orbifold theory is essential to describe tachyon condensation. This seems to be a generic feature since massless tachyon is always described by vertex operator with anti-periodic boundary condition. In our analysis, the twisted sectors actually do not play any rôle since we always sum over various sectors. I hope that these states may have some meanings to understand still mysterious Witten's $U(1)$ problem In superstring case, $Z_{2}$ operator $g$ is replaced by $(-1)^{F}$. Orbifold CFT is described by KT point.

\section{References}

[1] A. Sen, hep-th/9904207 and references therein.

[2] A. Sen, hep-th/9808141.

[3] A. Sen and B. Zwiebach, hep-th/9907164.

[4] M. Srednichi, JHEP 9808 (1998) 005, S. Sugimoto, hep-th/9905159.

[5] E. Witten, hep-th/9810188;

P. Horava, hep-th/9812135.

[6] O. Bergman, H. Gaberdiel, hep-th/9806155;

O. Bergman, H. Gaberdiel, hep-th/9908126;

M. Frau, L. Gallot, A. Lerda, P. Strigazzi, hep-th/9903123;

P. Di Vecchia, Lecture given in this workshop, hep-th/9912275. 
[7] A. Sen, hep-th/9911116;

A. Sen and B. Zwiebach, hep-th/9912249.

[8] P. Yi, hep-th/9901159.

[9] Y. Matsuo, hep-th/9905159.

[10] S. Weinberg, Phys. Lett. 187B (1987) 278;

M. Douglas and B. Grinstein, Phys. Lett. 183B (1987) 52;

N. Marcus and A. Sagnotti, Phys. Lett. 188B (1987) 58.

[11] A. Sen, hep-th/9902105.

[12] J. L. Cardy, Nucl. Phys. B324 (1989) 581.

[13] M. Douglas, hep-th/9807235;

M. Douglas and B. Fiol, hep-th/9903031.

[14] M. Oshikawa, I. Affleck, cond-mat/9612187. 


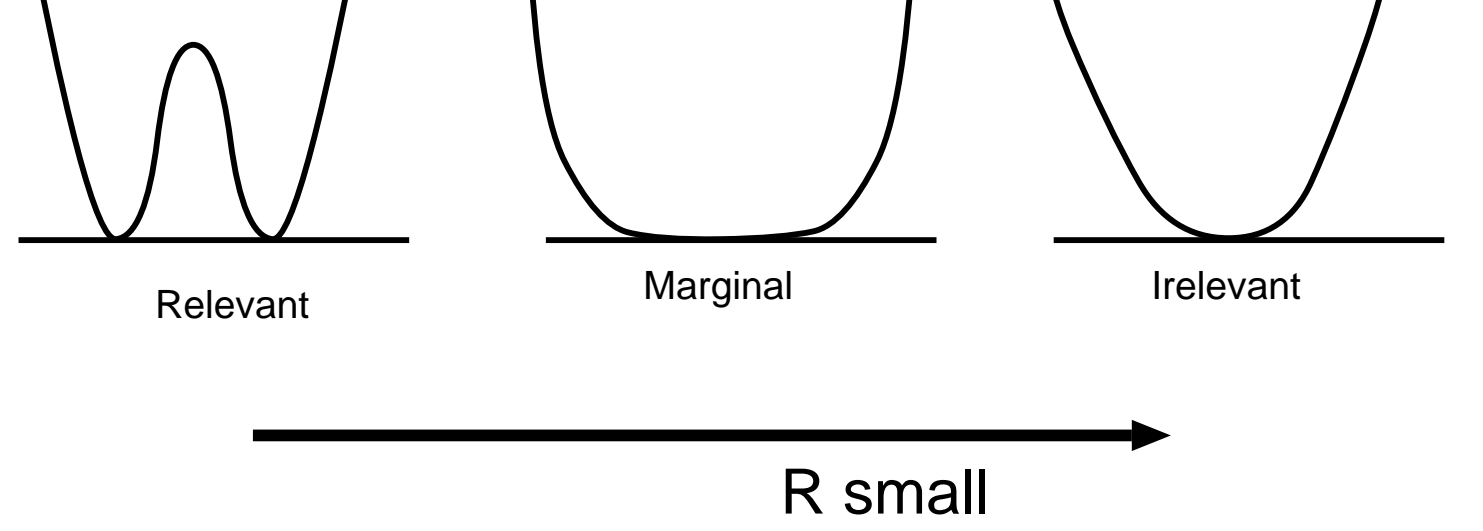




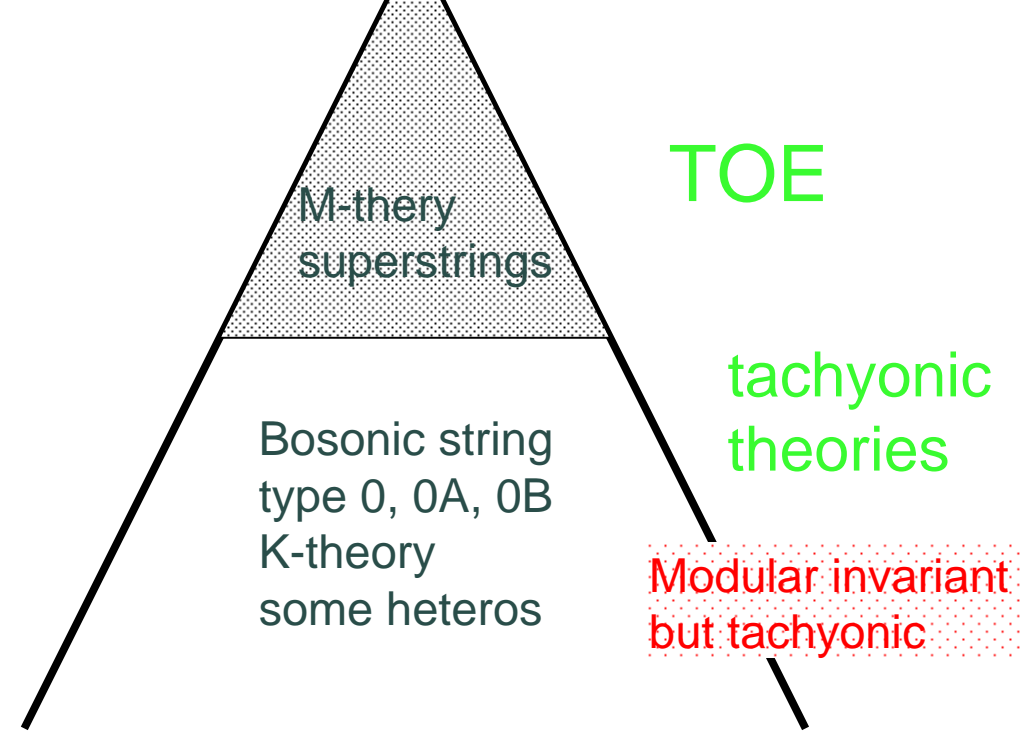

